\title{
Circadian Control of Membrane Excitability in Drosophila melanogaster Lateral Ventral Clock Neurons
}

\author{
Guan Cao and Michael N. Nitabach \\ Department of Cellular and Molecular Physiology, School of Medicine, Yale University, New Haven, Connecticut 06520
}

\begin{abstract}
Drosophila circadian rhythms are controlled by a neural circuit containing $\sim 150$ clock neurons. Although much is known about mechanisms of autonomous cellular oscillation, the connection between cellular oscillation and functional outputs that control physiological and behavioral rhythms is poorly understood. To address this issue, we performed whole-cell patch-clamp recordings on lateral ventral

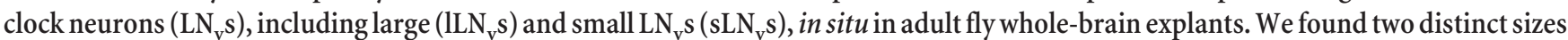
of action potentials (APs) in $>50 \%$ of $\mathrm{ILN}_{\mathrm{v}}$ s that fire APs spontaneously, and determined that large APs originate in the ipsilateral optic lobe and small APs in the contralateral. $\mathrm{LLN}_{\mathrm{v}}$ resting membrane potential (RMP), spontaneous AP firing rate, and membrane resistance are cyclically regulated as a function of time of day in $12 \mathrm{~h} \mathrm{light/dark} \mathrm{conditions} \mathrm{(LD).}$ LLN $_{\mathrm{v}}$ RMP becomes more hyperpolarized as time progresses from dawn to dusk with a concomitant decrease in spontaneous AP firing rate and membrane resistance. From dusk to dawn, $\mathrm{ILN}_{\mathrm{v}} \mathrm{RMP}$ becomes more depolarized, with spontaneous AP firing rate and membrane resistance remaining stable. In contrast, circadian defective per $^{0}$ null mutant ${ }^{1 L N_{v}}$ membrane excitability is nearly constant in LD. Over $24 \mathrm{~h}$ in constant darkness (DD), wild-type ILN $_{\mathrm{v}}$ membrane excitability is not cyclically regulated, although RMP gradually becomes slightly more depolarized. $\mathrm{SLN}_{\mathrm{v}} \mathrm{RMP}$ is most depolarized around lights-on, with substantial variability centered around lights-off in LD. Our results indicate that $\mathrm{LN}_{\mathrm{v}}$ membrane excitability encodes time of day via a circadian clock-dependent mechanism, and likely plays a critical role in regulating Drosophila circadian behavior.
\end{abstract}

Key words: circadian rhythms; Drosophila; membrane excitabiliy; time of day; clock neuron; cellular oscillation

\section{Introduction}

Drosophila circadian rhythms are governed by $\sim 150$ autonomously oscillating clock neurons, which include a number of anatomically and functionally distinct cell groups (HelfrichForster, 2003; Shafer et al., 2006; Nitabach and Taghert, 2008). Transcriptional feedback loops essential for cellular oscillation of clock neurons have been studied extensively and are well understood (for review, see Harmer et al., 2001; Hardin, 2005; Rosato et al., 2006). Cellular oscillation also relies on depolarizationdependent events at the clock neuron plasma membrane (Nitabach et al., 2002, 2005; Wu et al., 2008). However, the connection between cellular oscillation and clock neuron outputs that regulate Drosophila behavioral and physiological rhythms is still mostly unclear. The neuropeptide pigment dispersing factor (PDF)-expressing lateral ventral subset $\left(\mathrm{LN}_{\mathrm{v}}\right)$ of clock neurons has been proved to contain the pacemaker of the circadian control circuit in constant darkness (DD), and regulated release of PDF from small subset of $\mathrm{LN}_{\mathrm{v}}\left(\mathrm{sLN}_{\mathrm{v}}\right)$ nerve terminals plays a critical role in synchronizing downstream clock neurons (Renn et

Received 0ct. 18, 2007; revised May 9, 2008; accepted May 12, 2008.

This work was supported in part by the Whitehall Foundation and National Institute of Neurological Disorders and Stroke Grants R01NS055035 and R01NS056443 (M.N.N.). We thank Dr. Reiko Fitzimonds for the gift of equipments. We are grateful to members of the Nitabach Laboratory for their support and help, especially Dr. Ying Wu for her critical comments and discussion.

Correspondence should be addressed to Dr. Michael N. Nitabach, Department of Cellular and Molecular Physiology, School of Medicine, Yale University, New Haven, CT 06520. E-mail: michael.nitabach@yale.edu.

DOI:10.1523/JNEUROSCI.1503-08.2008

Copyright $\odot 2008$ Society for Neuroscience $\quad$ 0270-6474/08/286493-09\$15.00/0 al., 1999; Helfrich-Forster et al., 2000; Park et al., 2000; Peng et al., 2003; Stoleru et al., 2004; Nitabach et al., 2006). The molecular and cellular mechanisms of regulated release of PDF by $\mathrm{LN}_{\mathrm{v}} \mathrm{s}$ remain unclear.

Clock neurons of the mammalian suprachiasmatic nucleus (SCN) exhibit circadian time-dependent electrical properties, with a higher spontaneous AP firing rate and more depolarized resting membrane potential (RMP) during the day than at night (Green and Gillette, 1982; Groos and Hendriks, 1982; de Jeu et al., 1998; Kuhlman and McMahon, 2004). Clock-driven rhythms in various ionic conductances play key roles in circadian regulation of RMP and spontaneous AP frequency in mammalian clock neurons (de Jeu et al., 1998; Pennartz et al., 2002; Kuhlman and McMahon, 2004; Itri et al., 2005; Pitts et al., 2006). However, very little is known about the regulation of membrane excitability of Drosophila clock neurons.

Park and Griffith (2006) reported the first whole-cell recordings from the large subset of $\mathrm{LN}_{\mathrm{v}} \mathrm{s}\left(\mathrm{LN}_{\mathrm{v}} \mathrm{s}\right)$ in whole-brain explants, and compared $\mathrm{ILN}_{\mathrm{v}} \mathrm{RMP}$ and membrane resistance at two times of day in light/dark conditions (LD) and DD. Although they failed to detect spontaneous APs in their recordings, raising the possibility that their recording conditions could be different from in vivo physiological conditions, they found that time of day and lighting condition affected $\mathrm{lLN}_{\mathrm{v}} \mathrm{RMP}$ and membrane resistance. This study is consistent with recent work of the Sheeba et al. (2008), who showed rhythmic changes in $\mathrm{ILN}_{\mathrm{v}} \mathrm{RMP}$ and AP firing rate assayed at four different time points after 2 weeks in 
constant darkness. We have developed an optimized recording protocol that reveals spontaneous activity and likely more closely replicates physiological conditions, and used this protocol to investigate the modulation of $\mathrm{LN}_{\mathrm{v}}$ membrane properties continuously across the $24 \mathrm{~h}$ day. Applying this approach to wild-type (WT) and circadian-defective mutant flies, we show that $\mathrm{ILN}_{\mathrm{v}}$ clock neurons encode time of day via circadian clock-dependent oscillatory modulation of membrane excitability. We also apply this approach to the very difficult-to-record-from $\mathrm{sLN}_{\mathrm{v}} \mathrm{s}$, demonstrating that their RMP is most depolarized around lights-on in LD.

\section{Materials and Methods}

Fly stocks. Flies were maintained at $25^{\circ} \mathrm{C}$ in a $12 \mathrm{~h}$ light/dark cycle with $\sim 2000$ lux in the light phase. Female pdf-GAL4/CyO;UAS-RFP/ $T M 6 C, S b$ flies that express red fluorescent protein (RFP) solely in $\mathrm{LN}_{\mathrm{V}}$ clock neurons were collected for electrophysiological recordings. Virgin female $y \operatorname{per}^{0} w$ flies were crossed with male $p d f-G A L 4 / C y O ; U A S-R F P /$ $T M 6 C, S b$ flies for collecting male $y$ per $^{0} w ; p d f-G A L 4 /+; U A S-R F P /+$ flies for electrophysiological experiments.

Brain dissection. After eclosion (3-7 d), flies were anesthetized with $\mathrm{CO}_{2}$ and then immersed in $70 \%$ ethanol for $\sim 30 \mathrm{~s}$ before being transferred to a dissection chamber filled with external recording solution. External solution consisted of (in mM) $101 \mathrm{NaCl}, 3 \mathrm{KCl}, 1 \mathrm{CaCl}_{2}, 4$ $\mathrm{MgCl}_{2}, 1.25 \mathrm{NaH}_{2} \mathrm{PO}_{4}, 5$ glucose, and $20.7 \mathrm{NaHCO}_{3}$, pH 7.2, with an osmolarity of $250 \mathrm{mmol} / \mathrm{kg}$. After head cuticle, eyes, proboscis, and trachea were removed with fine forceps, the brain was dissected out and placed on the floor of the recording chamber, ventral side up. A mammalian brain slice "harp" holder with a $0.5 \mathrm{~mm}$ interval between each nylon fiber was placed on top of the fly brain to secure it during recording.

Electrophysiology. Fly brains were continuously perfused with external solution bubbled with $95 \% \mathrm{O}_{2} / 5 \% \mathrm{CO}_{2}$ at room temperature. $\mathrm{LN}_{\mathrm{v}} \mathrm{s}$ were visualized by RFP fluorescence using an Olympus fixed-stage upright microscope (BX50WI); $s \mathrm{LN}_{\mathrm{V}} \mathrm{s}$ and $\mathrm{LN}_{\mathrm{V}} \mathrm{s}$ were distinguished by their sizes and anatomical locations. The immediate area surrounding the intended $\mathrm{lLN}_{\mathrm{V}}$ or $\mathrm{sLN}_{\mathrm{v}}$ was enzymatically digested with focal application of protease XIV ( $2 \mathrm{mg} / \mathrm{ml}$; Sigma). Whole-cell recordings were performed using borosilicate standard wall capillary glass pipettes (Sutter Instrument) and data were acquired with Axopatch 200B amplifier, Digidata 1200 A/D hardware, and pClamp 8.0 software (Molecular Devices). Recording pipettes are filled with internal solution consisting of (in $\mathrm{mM}$ ) 102 potassium gluconate, $17 \mathrm{NaCl}, 0.085 \mathrm{CaCl}_{2}, 4 \mathrm{Mg}$-ATP, $0.5 \mathrm{Na}-\mathrm{GTP}$, 0.94 EGTA, and 8.5 HEPES, pH 7.2, with an osmolarity of $235 \mathrm{mmol} / \mathrm{kg}$. Gigaohm seals were achieved before recording in cell-attached configuration in voltage-clamp mode, followed by break-in to whole-cell configuration while in voltage-clamp mode. To confirm maintenance of a good seal and absence of damage to the cell, a $40 \mathrm{mV}$ hyperpolarizing pulse was imposed on each cell while in whole-cell voltage-clamp mode from a holding potential of $-80 \mathrm{mV}$. Only if the resulting inward leak current was less than $-50 \mathrm{pA}$ was that cell used for subsequent currentclamp measurements of RMP, AP firing rate, and membrane resistance. RMP was determined after stabilization of the membrane potential after the transition from voltage-clamp to current-clamp mode, and for cells with oscillating membrane potential was defined at the trough of the oscillation. AP firing rate was computed over the 5 min period after the transition from voltage-clamp to current-clamp configuration. Membrane resistance was measured by injecting $-20 \mathrm{pA}$ current in currentclamp recording mode. The amplitude of sinusoidal oscillation of RMP was defined as the difference between peak and trough. For recordings on flies in the dark phase, all recordings were made within $20 \mathrm{~min}$ from the fly being taken out of the incubator to minimize any acute effect of light.

For the gap junction inhibitor experiments, halothane, carbenoxolone (CBX), and 2-octanol were purchased from Sigma-Aldrich. Halothane and CBX were prepared with external solution, and 2-octanol was dissolved in DMSO and diluted with external solution.

Statistical analysis. Relationships between time of day and parameters of membrane electrical excitability were modeled using linear regression
(Origin 6.1; OriginLab) and nonlinear regression analysis (SPSS 14). The null hypothesis we tested in linear regression analysis is that the slope of any fitted regression line is zero. For nonlinear regression analysis, the temporal relationship between $1 \mathrm{LN}_{\mathrm{v}} \mathrm{RMP}$ and time of day was fitted to a sinusoidal curve. The null hypothesis for this analysis is that there is no sinusoidal relationship between RMP and time of day. We modeled $\mathrm{LLN}_{\mathrm{v}}$ $\mathrm{RMP}$ as a function of time of day (ZT) in LD: RMP $=a \times \sin (b+c \times \mathrm{ZT})$ $+d$.

The period was calculated using the formula period (hours) $=2 \pi / c$, and the peak and trough of the sinusoid curve were defined as peak (hours) $=(\pi / 2-b) / c$ and trough (hours) $=(3 \pi / 2-b) / c$, respectively. Best fits to the data were generated using the Sequential Quadratic Programming iterative fitting algorithm, with initial parameters of $a=-0.1$, $b=-0.1, c=-0.1$, and $d=-0.1$. These initial parameter values represent a very shallow sinusoid with a very long period. To test the validity of fits to the real data, we fit randomly reshuffled data sets generated by reshuffling the RMP associated with each time point measured. We combined both linear regression analysis and nonlinear regression analysis to further validate our sinusoidal model. We analyzed the data between the peak and trough of the fitted sinusoidal curve with linear regression analysis. If the null hypothesis was accepted in linear regression analysis (i.e., RMP distributed like a horizontal line from peak to trough), we then accepted the null hypothesis in nonlinear regression analysis even if the $p$ value in nonlinear regression analysis was $<0.05$ (i.e., RMP versus time of day did not fit a sinusoidal model). The rationale is that the linear fit of data from the peak to trough of a sinusoidal curve should be significantly different from a horizontal line.

\section{Results}

\section{Characterization of membrane excitability of $\mathrm{ILN}_{\mathrm{v}}$} clock neurons

We performed whole-cell electrophysiological recordings on $\mathrm{ILN}_{\mathrm{v}}$ clock neurons and characterized RMP, spontaneous AP firing rate, and membrane resistance. To verify that our internal recording solution was reasonably close to physiological, we first recorded spontaneous AP firing in cell-attached configuration after forming a gigaohm seal but before breaking into whole-cell configuration. This allowed us to record APs without dialyzing the cytoplasm with pipette solution. One example of such a recording is shown in Figure $1 \mathrm{~A}$. During $3 \mathrm{~min}$ in cell-attached configuration, this cell exhibited 31 bursts of APs, with each burst containing two to six APs. After breaking into whole-cell currentclamp configuration, 52 bursts of APs occured in 5 min. The AP firing frequencies and patterns were very similar both before and after breaking into whole-cell configuration, suggesting that our recording solutions permit reasonably physiological spontaneous membrane activity.

Not surprisingly, there is some variability from cell to cell in $1 \mathrm{LN}_{\mathrm{v}}$ membrane activity. As shown in the $10 \mathrm{~s}$ trace in Figure $1 \mathrm{~A}$, this $\mathrm{LLN}_{\mathrm{v}}$ exhibits $0.2 \mathrm{~Hz} \sim 10 \mathrm{mV}$ oscillation in RMP, with a burst of two to six APs riding on the depolarized phase. In another cell shown in Figure $1 B$, RMP oscillates with an $\sim 5 \mathrm{mV}$ amplitude, and spontaneous APs occur on the depolarized phase of some RMP cycles, but not the majority of them (82\%). Some $1 \mathrm{LN}_{\mathrm{v}} \mathrm{s}$ exhibit two different sizes of APs (Fig. 1C). We categorized the spontaneous membrane activity during the light phase of LD of $58 \mathrm{lLN}_{\mathrm{v}} \mathrm{s}$ from $>50$ different fly brains. Thirty-five $\mathrm{lLN}_{\mathrm{v}} \mathrm{s}$ exhibited RMP oscillation with an average frequency of $0.28 \pm 0.18 \mathrm{~Hz}$ and an average peak-to-trough amplitude of $7.4 \pm 3.5 \mathrm{mV}$ (mean $\pm \mathrm{SD})$. Neither the frequency nor amplitude of this oscillation is significantly correlated to time of day (linear regression, $p>0.05)$. Of these 35 oscillating cells, 26 exhibited spontaneous AP bursts, whereas 9 were silent. There was no correlation between spontaneous AP firing and amplitude of RMP oscillation ( $p>0.05)$. Of the 23 nonoscillating cells, six exhibited repetitive 
A
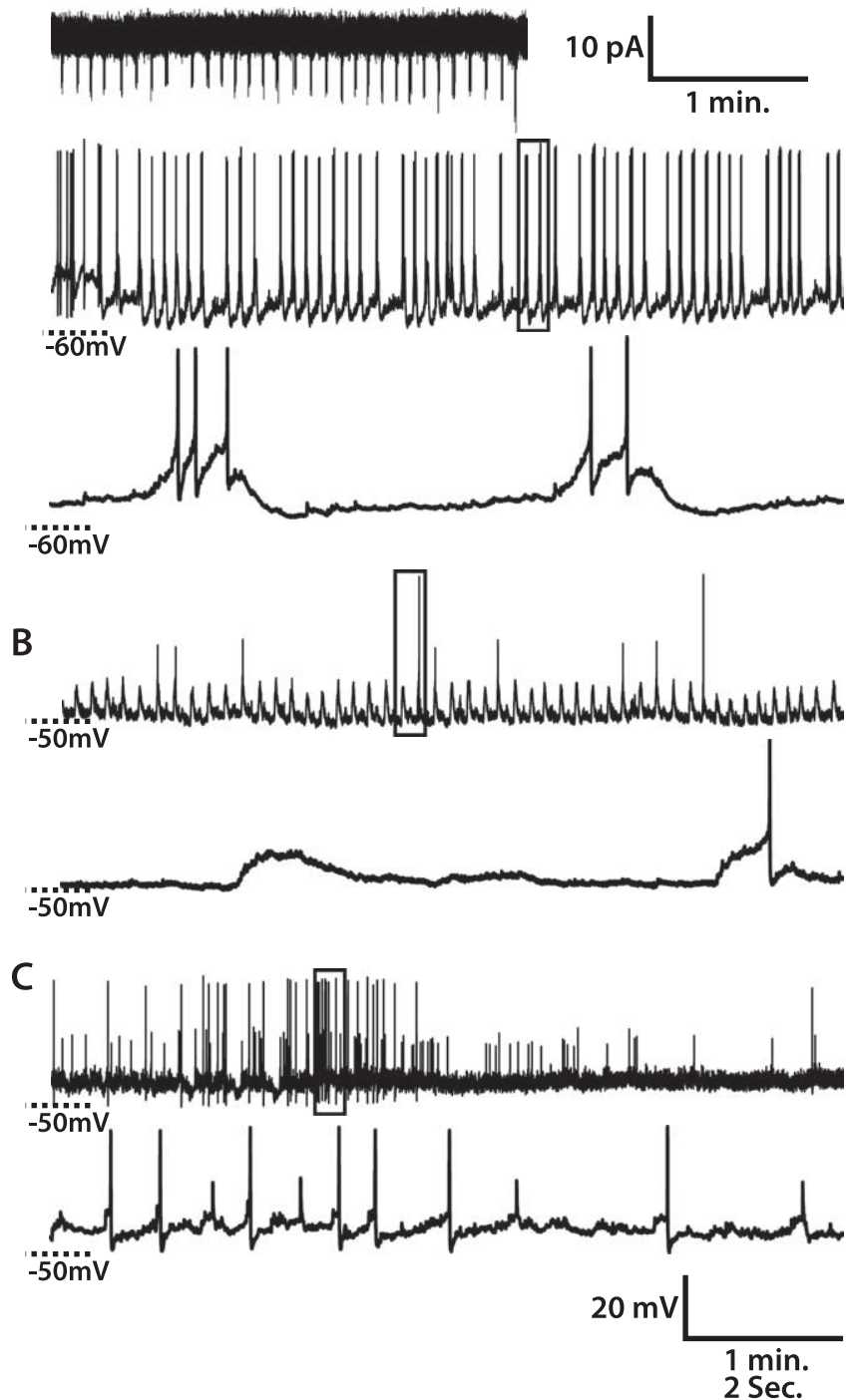

Figure 1. Spontaneous activity of WT ILN $\mathrm{N}_{\mathrm{v}}$ clock neurons. $A-C$, Three examples of whole-cell recordings from ILN $\mathrm{N}_{v}$. $A$, Top, A 3 min recording in cell-attached configuration. The middle panel in $\boldsymbol{A}$ and top panels in $\boldsymbol{B}$ and $\boldsymbol{C}$ are 5 min recordings in whole-cell current-clamp configuration. The bottom panels depict magnified views of the regions of the respective 5 min recordings outlined in the frame. For the $I L N_{v}$ in $A$, spontaneous AP firing rates and patterns are similar in cell-attached and in whole-cell configurations. ILN ${ }_{v}$ RMP oscillates, and bursts of spontaneous APs occur on the depolarized phase of the RMP oscillations. For the ILN $\mathrm{N}_{v}$ in $\boldsymbol{B}$, RMP oscillates, and spontaneous APs occur on the depolarized phase of some RMP oscillations but not the majority of them. For the ILN $\mathrm{v}_{\mathrm{v}}$ in $\mathrm{C}$, RMP does not oscillate and two different sizes of APs occur.

sustained high-frequency bursts, three exhibited tonic highfrequency firing, five fired APs without any obvious pattern, and seven were silent. Of the 38 total cells that fired APs, 16 had APs all of approximately the same magnitude, peaking at $\sim 45 \mathrm{mV}$ above rest, whereas 22 also had a second population of APs peaking only $\sim 20 \mathrm{mV}$ above rest. There was no relationship between time of day and the presence or absence of RMP oscillations or multiple AP sizes.

Small action potentials come from the contralateral optic lobe

Because there are two distinct sizes of APs in $>50 \%$ of $1 \mathrm{LN}_{\mathrm{v}} \mathrm{s}$ that fire APs, we investigated the cellular mechanisms of these two different APs. $\mathrm{LLN}_{\mathrm{v}} \mathrm{s}$ extend ramified arborization in the optic lobes of both sides of the brain, and dendritic aborizations of all
$5 \mathrm{~min}$ before $0.1 \%$ Halothane

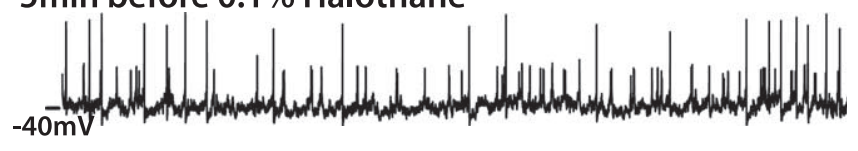

$3 \min$ in $0.1 \%$ Halothane

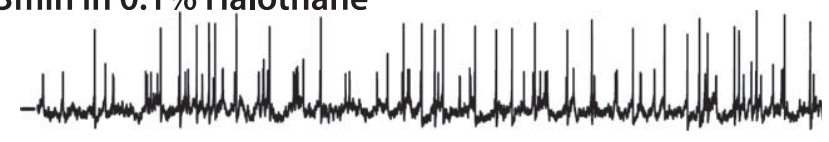

$12 \mathrm{~min}$ in $0.1 \%$ Halothane

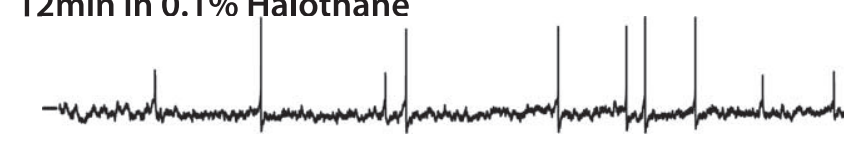

$3 \mathrm{~min}$ after washout

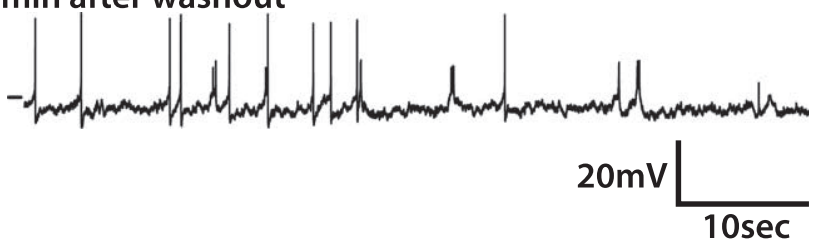

Figure 2. Gap junction inhibitors do not affect the amplitude of small APs. One example showing the effect of gap junction inhibitor, halothane (0.1\%), on APs in ILN $\mathrm{L}_{v}$ is shown. The $\mathrm{ILN}_{\mathrm{v}}$ is incubated in external solution containing $0.1 \%$ halothane for $20 \mathrm{~min}$ before washout with normal external solution. Several 1 min traces before and after the application of halothane and after washout are shown here. The frequency but not amplitude of small APs decreases after $10 \mathrm{~min}$ treatment of halothane.

$1 \mathrm{LN}_{\mathrm{v}} \mathrm{s}$ from either side of the fly brain mostly overlap (for review, see Helfrich-Forster, 2002, 2003). This makes it possible that lL$\mathrm{N}_{\mathrm{v}} \mathrm{s}$ are electrically coupled with each other via gap junctions, and small APs arise from large APs in neighboring $\mathrm{lLN}_{\mathrm{v}} \mathrm{s}$ propagating to the recorded cell through gap junctions. Also, each $\mathrm{LLN}_{\mathrm{v}}$ contains dendritic arbors in each of the two optic lobes (Park and Griffith, 2006). APs generated in the contralateral optic lobe travel a significantly longer distance $(300-500 \mu \mathrm{m})$ than those generated in the ipsilateral optic lobe $(<50 \mu \mathrm{m})$ to reach the soma, where signals are recorded. Therefore, another possibility is that small APs result from the decay of large APs generated in the contralateral optic lobe as they travel passively along the posterior optic tract (POT) to reach the $\mathrm{ILN}_{\mathrm{v}}$ soma.

To test the electrical coupling hypothesis, we examined whether pharmacological gap junction inhibitors could specifically reduce the magnitude of small APs. Three different gap junction inhibitors, halothane (0.1\%), 2-octanol (2 mM), and carbenoxolone (CBX, $1 \mathrm{~mm}$ ) (Schneider and Stengl, 2006), were used. All three gap junction inhibitors decreased the frequency of small APs, but had no effect on the amplitude. Gap junction inhibitors showed a complex effect on large APs. Like small APs, the amplitude of large APs was unaltered by gap junction inhibitors; however, the frequency of large APs exhibited mixed responses, as five of eight $l \mathrm{LN}_{\mathrm{v}} \mathrm{s}$ in halothane, one of four in CBX, and one of five in 2-octanol showed an increase, whereas three in halothane, two in CBX, and four in 2-octanol showed a decrease, and one in CBX showed no change. In the example shown in Figure 2, after the application of $0.1 \%$ halothane, both large and small AP firing frequencies decreased gradually but were not totally abolished. Importantly, neither large nor small AP amplitude was changed by halothane. Bath application of gap junction blockers is expected to induce a gradual decrease in the amplitude of small APs as the blockade takes effect if they were propagating from coupled cells through gap junctions. Because only the frequency but not the amplitude of small APs decreased after block- 
ing gap junctions, it is unlikely that small APs are generated in coupled cells and propagated through gap junctions to the cell being recorded from.

We then tested the hypothesis that small APs originate in the distal contralateral arbor of the cell being recorded from. As depicted schematically in Figure $3 A$, we disconnected $1 \mathrm{LN}_{\mathrm{v}}$ soma from their contralateral arbors by severing the POT in the middle of the brain using a scalpel. As a sham-cut control, we made a similar cut, but dorsal to the POT. After cutting the POT, only large APs were found in all $\mathrm{LLN}_{\mathrm{v}}$ s tested $(n=7)$, whereas after the sham operation of eight $\mathrm{lLN}_{\mathrm{v}} \mathrm{s}$, one was silent, two exhibited only large APs, and five showed both large and small APs. We also cut some fly brains into two complete halves along the midline. Of 19 $1 \mathrm{LN}_{\mathrm{v}} \mathrm{s}$ recorded from half brains, 5 did not fire any APs, 13 fired only large APs, and 1 fired both large and small APs. Unlike in the whole brain, where the occurrence of small APs appears temporally uncorrelated with large APs, every small AP recorded from the $\mathrm{lLN}_{\mathrm{v}}$ in the single half brain that exhibits small APs occurred $\sim 10$ ms before a large AP, suggesting these small APs may have a different origin from those recorded in the normal whole brain preparation. The likelihood of occurrence of small APs in $1 \mathrm{LN}_{\mathrm{v}} \mathrm{s}$ in cut-POT fly brains and complete half brains is significantly different from that in the control fly brains ( $\chi^{2}$ test, both comparisons, $p<0.0001)$. These results strongly suggest that small APs originate in the contralateral optic lobe arbor of the $\mathrm{ILN}_{\mathrm{v}}$ recorded from.

\section{Regulation of $\mathrm{ILN}_{\mathrm{v}}$ membrane excitability in LD}

We assessed the dependence of membrane excitability on time of day in $\mathrm{LD}$. We recorded $109 \mathrm{LLN}_{\mathrm{v}} \mathrm{s}$ at different times in LD, nearly all of them $(>100)$ from different individual fly brains. Figure 4 depicts scatter plots of RMP, spontaneous AP firing rate, and membrane resistance as a function of the time when recordings were performed, with each data point representing an individual $\mathrm{ILN}_{\mathrm{V}}$. In LD, RMP exhibits a robust cyclical dependence on time of day, highest just after lights-on, declining over the course of the day (linear regression, $r=-0.54 ; p<0.0001$ ), and then increasing during the night (linear regression, $r=0.41$; $p<0.01$ ). Consistent with this temporal pattern of RMP in the light phase, spontaneous AP firing rate is highest just after lights-on, and becomes lower as the day progresses (linear regression, $r=$ $-0.35 ; p<0.01)$. In the light phase, membrane resistance also decreases over time (linear regression, $r=-0.49$; $p<0.01$ ). In the dark phase, spontaneous AP firing rate and membrane resistance do not change significantly over time (linear regression, $p>0.1$.

We also fit the relationship between RMP and time of day to a sinusoidal model. RMP versus time of day best fits the sinusoidal model $y=6.0 \times \sin (1.75+0.29 \times x)-49.7$ (nonlinear regression, $p<0.0001$ ). The amplitude of this sinusoid is $6.0 \mathrm{mV}$ and the period is $21.4 \mathrm{~h}$. We also fit multiple randomly reshuffled data sets of RMP versus time of day to a sinusoidal model, and in all cases best fits were to sinusoids with very short noncircadian periods (supplemental Fig. 1, available at www.jneurosci.org as supplemental material). Data sets are randomized by randomly reshuffling the measured RMPs for each cell among the times of day of each recording. This absence of significant fit of any randomly reshuffled data sets to a circadian sinusoid strongly supports the validity of the sinusoidal fit to the real data. The peak and trough of the sinusoid of RMP versus time of day are indicated by vertical dashed lines in Figure 4 . The decrease in RMP between the peak and trough and the increase between the trough and peak of the sinusoid are both statistically significant by linear
A

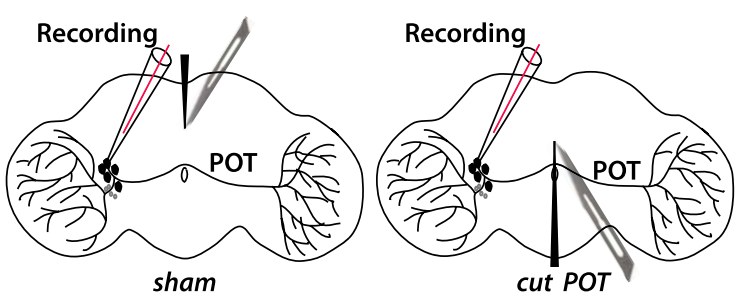

B

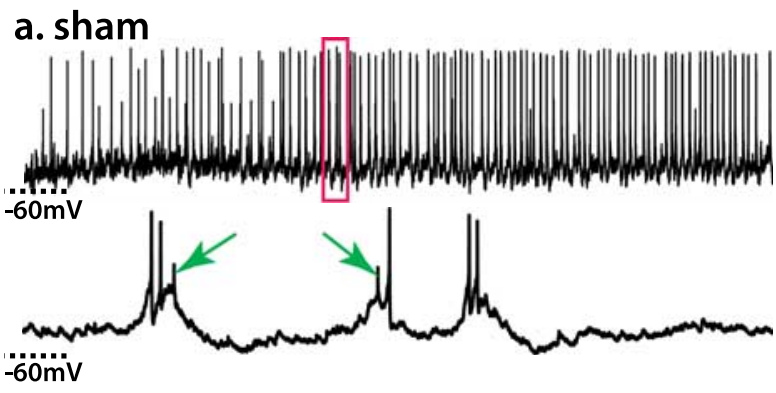

b. cut POT

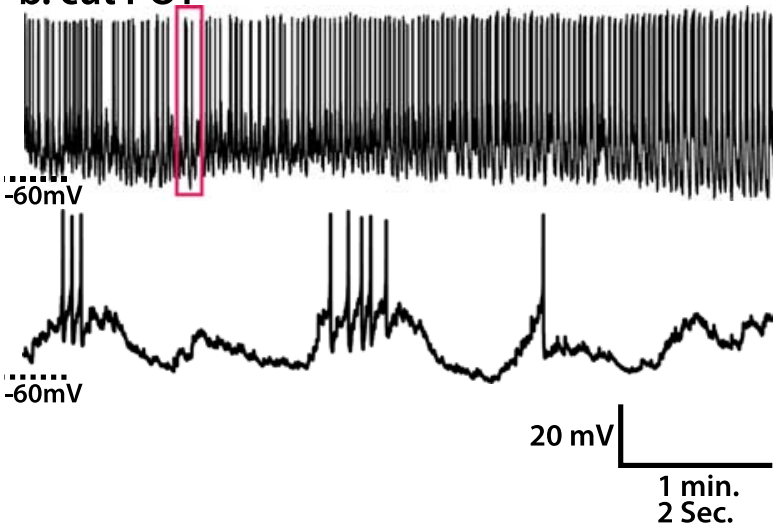

C

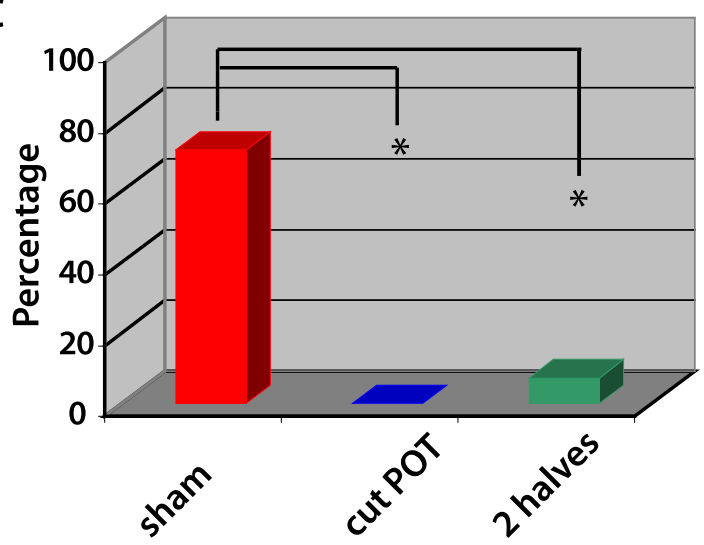

Figure 3. Small APs of ILN $\mathrm{N}_{\mathrm{v}}$ s originate in the contralateral optic lobe. $\boldsymbol{A}$, Schematic diagrams of experimental design to address the origin of small APs. The left diagram shows the sham operation, in which a cut is made at the dorsal aspect of the brain without damaging the POT. The right diagram shows the experimental operation, in which the POT is cut. $\boldsymbol{B}$, Representative traces recorded in the control and experimental group. In the control $(\boldsymbol{a})$, the sham operation does not eliminate small APs (indicated by arrows). In the experimental group (b), only large APs occur. C, Histogram summarizing the percentage of ILN $\mathrm{N}_{\mathrm{v}}$ that fire both small and large APs after sham operation (red), cutting the POT (blue), and completely separating the brain into two halves (green). One silent cell that did not fire any APs was excluded from this analysis. The sham operation group was significantly different from both experimental groups, cutting the POT, or completely separating two hemispheres ( $\chi^{2}$ test, both $\left.{ }^{*} p<0.005\right)$. 


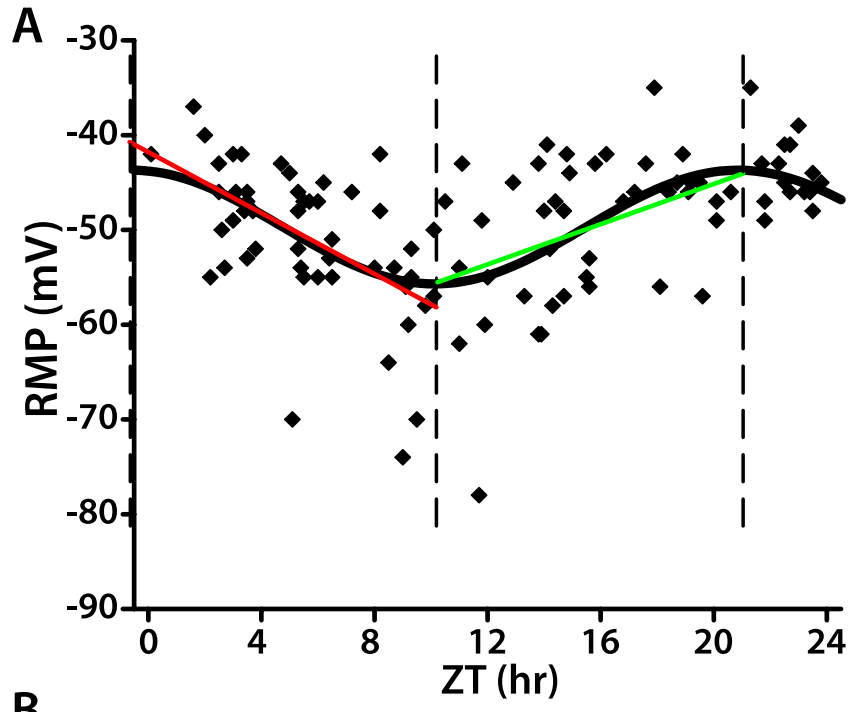

B
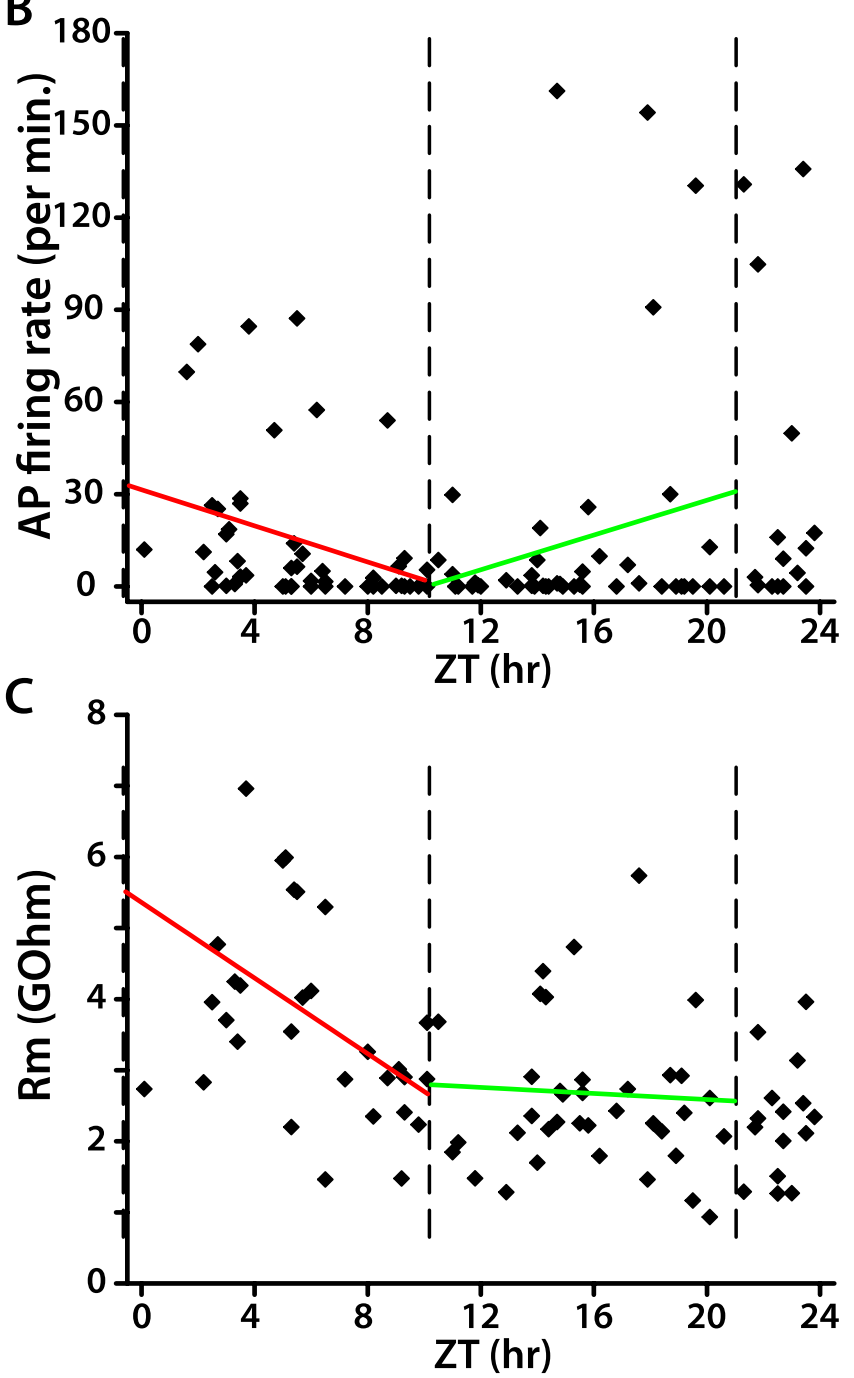

Figure 4. WT ILN $\mathrm{v}_{\mathrm{v}}$ membrane excitability in $L D$ is strongly rhythmic. $A-C$, Each data point in the scatter plots represents the RMP $(\boldsymbol{A})$, spontaneous AP firing rate $(\boldsymbol{B})$, or membrane resistance $(\boldsymbol{C})$ of one ILN $\mathrm{N}_{\mathrm{v}}$ at the indicated time of recording. In $\boldsymbol{A}$, the sinusoidal curve represents the best fit of RMP as a function of time of day in LD (ZT, nonlinear regression; $p<0.0001$ ). The three vertical dashed lines indicate the peak and trough of the fitted sinusoid of RMP versus ZT. Red and green lines depict the linear regression analysis of data within those two intervals $(\boldsymbol{A}$ both red and green linear fits, $p<0.01 ; B, C$, red linear fit, $p<0.05$; green linear fit, $p>0.1$ ). regression analysis $(p<0.01)$. Spontaneous AP firing rate and membrane resistance decrease significantly from the peak to trough of the sinusoid (linear regression, $p<0.05$ ). However, there is no statistically significant change in spontaneous AP firing rate and membrane resistance over time from the trough to peak $(p>0.1)$. These different methods of statistical analysis all strongly support the conclusion that time of day in LD is encoded by $\mathrm{ILN}_{\mathrm{v}}$ membrane excitability.

Regulation of $\operatorname{per}^{O}$ circadian mutant $\mathrm{ILN}_{\mathrm{v}}$ membrane excitability in LD

To test whether rhythmic changes in WT $\operatorname{lLN}_{\mathrm{v}}$ membrane excitability are simply responses to the LD cycle (i.e., "masking") or, alternatively, depend on circadian clock function, we assayed membrane excitability of $1 \mathrm{LN}_{\mathrm{v}} \mathrm{s}$ in per $^{0}$ null mutant flies. per ${ }^{0}$ flies lack a functional circadian clock, exhibit only behavioral masking responses to light transitions in LD, and are arrhythmic in DD (supplemental Fig. 3, available at www.jneurosci.org as supplemental material) (Konopka and Benzer, 1971). We recorded 87 $\mathrm{LLN}_{\mathrm{v}} \mathrm{s}$ from per $^{\circ}$ flies in LD, each from one individual fly. The distribution of $\operatorname{per}^{0} 1 \mathrm{LN}_{\mathrm{v}} \mathrm{RMP}$ either in the light phase, in the dark phase, or over the entire $24 \mathrm{~h}$ cycle is not significantly different from a horizontal line (linear regression, $p>0.1$ ). The relationship between $\operatorname{per}^{0} \mathrm{ILN}_{\mathrm{v}} \mathrm{RMP}$ and time of day best fits a sinusoidal model $y=-1.4 \times \sin (3.39-0.26 \times x)-44.8(p<0.0001)$ (Fig. $5 A$ ). Whereas the fit to this sinusoid is statistically significant, its amplitude is less than one-fourth that of WT (compare Fig. 4). Randomly reshuffled data sets of per ${ }^{0} \mathrm{ILN}_{\mathrm{v}}$ RMP versus time of day do not fit sinusoids with circadian period (supplemental Fig. 2, available at www.jneurosci.org as supplemental material), suggesting that the small-amplitude circadian oscillation observed in the raw data is valid. However, linear regression analysis of RMP from the trough to peak of the sinusoid reveals no significant relationship between RMP and time of day $(p>0.1)$. Linear regression analysis between lights-on and the trough of the sinusoid reveals that RMP becomes slightly more hyperpolarized during this interval $(p<0.05)$. Correspondingly, per $^{0} \mathrm{ILN}_{\mathrm{v}}$ membrane resistance decreases gradually over this interval $(p<0.05)$, but spontaneous AP firing rate is not significantly changed $(p>$ 0.05). The RMP, spontaneous AP firing rate, and membrane resistance of $\operatorname{per}^{0} 1 \mathrm{LN}_{\mathrm{v}} \mathrm{s}$ do not change significantly after this $7 \mathrm{~h}$ interval starting at lights-on, or when analyzed for the entire light phase, dark phase, or complete $24 \mathrm{~h}$ cycle (linear regression, $p>$ 0.1 ). Therefore, these brief, time-dependent changes in $\operatorname{per}^{\circ} \operatorname{lLN}_{\mathrm{v}}$ membrane excitability right after lights-on in LD must be masking effects, because per $^{\circ}$ flies have no circadian timekeeper. We therefore conclude that the much larger and more robust timedependent membrane excitability changes observed in WT $1 \mathrm{LN}_{\mathrm{v}} \mathrm{s}$ in LD almost wholly arise out of circadian clock function.

\section{WT ILN $_{\mathrm{v}}$ membrane excitability in DD}

Rhythmic transcriptional feedback ceases in $\operatorname{LLN}_{\mathrm{v}} \mathrm{s}$, but not other clock neurons, by the first day after transition from LD to DD (Yang and Sehgal, 2001; Shafer et al., 2002). Thus, if robust clockdependent oscillation of $\mathrm{ILN}_{\mathrm{v}}$ membrane excitability in LD is cell autonomous (i.e., driven by cellular oscillation of the $1 \mathrm{LN}_{\mathrm{v}} \mathrm{s}$ themselves), then the prediction is that $\mathrm{ILN}_{\mathrm{v}}$ membrane excitability will not oscillate in DD. To test this prediction, we measured $\mathrm{ILN}_{\mathrm{v}}$ membrane excitability in DD. As shown in Figure $6, \mathrm{lLN}_{\mathrm{v}}$ RMP becomes slightly more depolarized over time in DD (linear regression, slope, $0.14 ; p<0.01$ ), but there is no hint of any circadian rhythm, as revealed by the inability to fit these data to a sinusoid with circadian period. Spontaneous AP firing rate and 

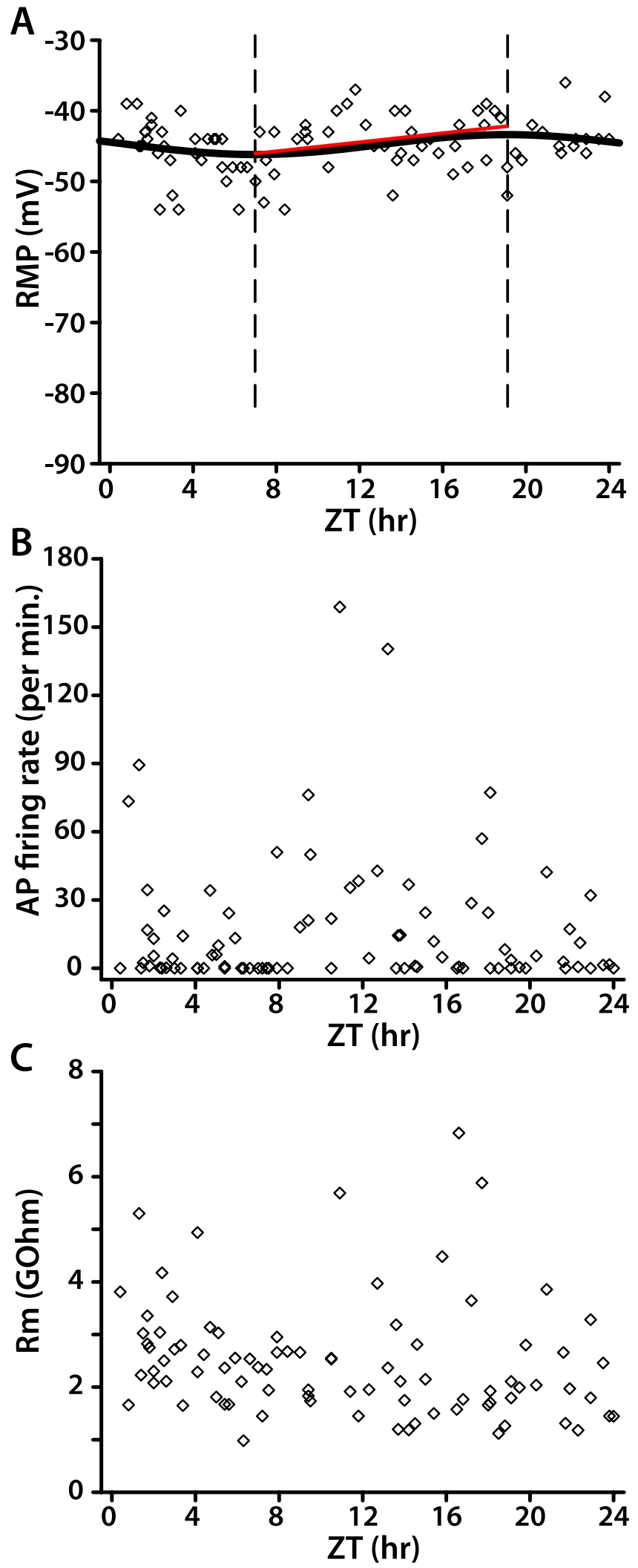

Figure 5. $\operatorname{per}^{0} \mathrm{ILN} \mathrm{N}_{v}$ membrane excitability in $\mathrm{LD}$ is only very weakly rhythmic. $A-C$, Scatter plots of per $^{0} I \operatorname{LN}_{v} \operatorname{RMP}(\boldsymbol{A})$, spontaneous AP firing rate $(\boldsymbol{B})$, and membrane resistance $(\boldsymbol{C})$. In $\boldsymbol{A}$, the sinusoid represents the best fit of RMP versus time of day (nonlinear regression analysis, $p<0.0001$ ), two vertical dashed lines depict the trough and peak of the sinusoidal curve, and the red line is the linear regression analysis of the data between the trough and peak $(p>0.1)$.
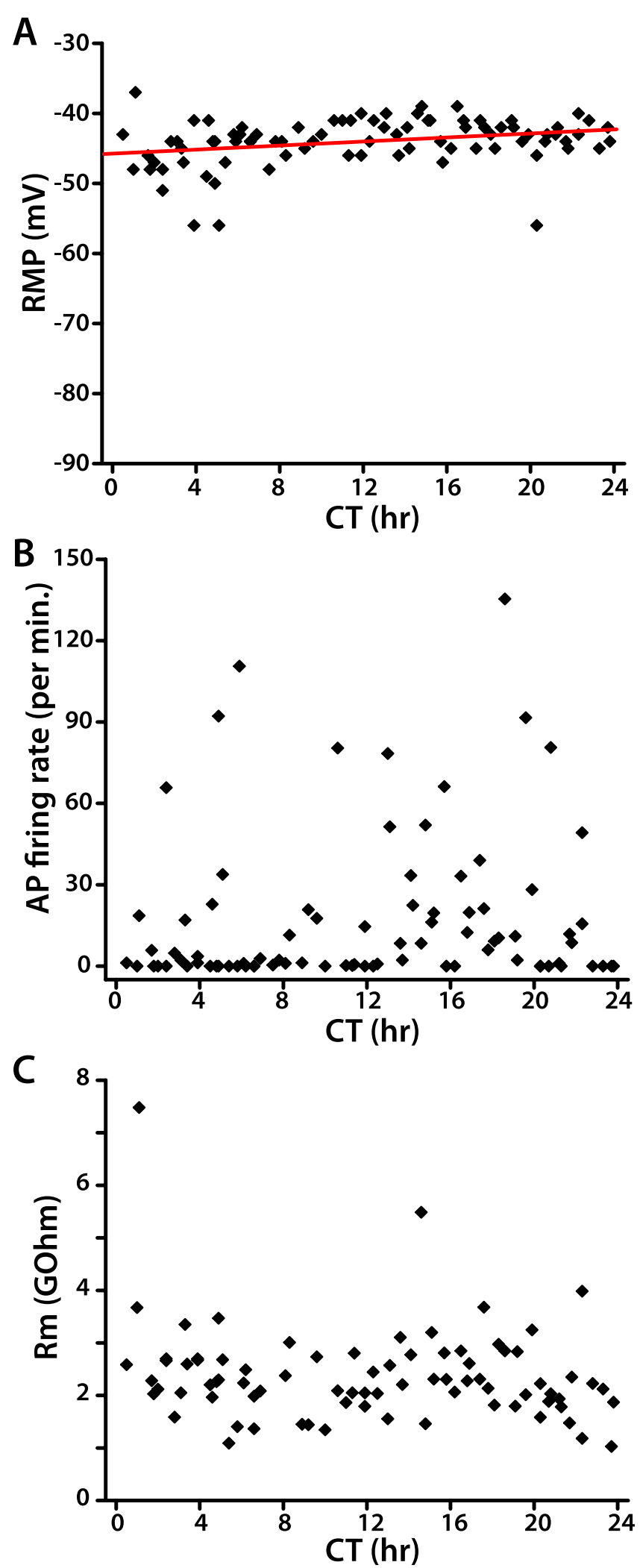

Figure 6. WT ILN $\mathrm{v}_{\mathrm{v}}$ membrane excitability in DD is not rhythmic. $A-C$, Scatter plots of WT ILN $\operatorname{RMP}(\boldsymbol{A})$, spontaneous AP firing rate $(\boldsymbol{B})$, and membrane resistance $(\boldsymbol{C})$. In $\boldsymbol{A}$, the red line depicts the linear regression analysis of the relationship between RMP and time of day $(p<0.05)$. 

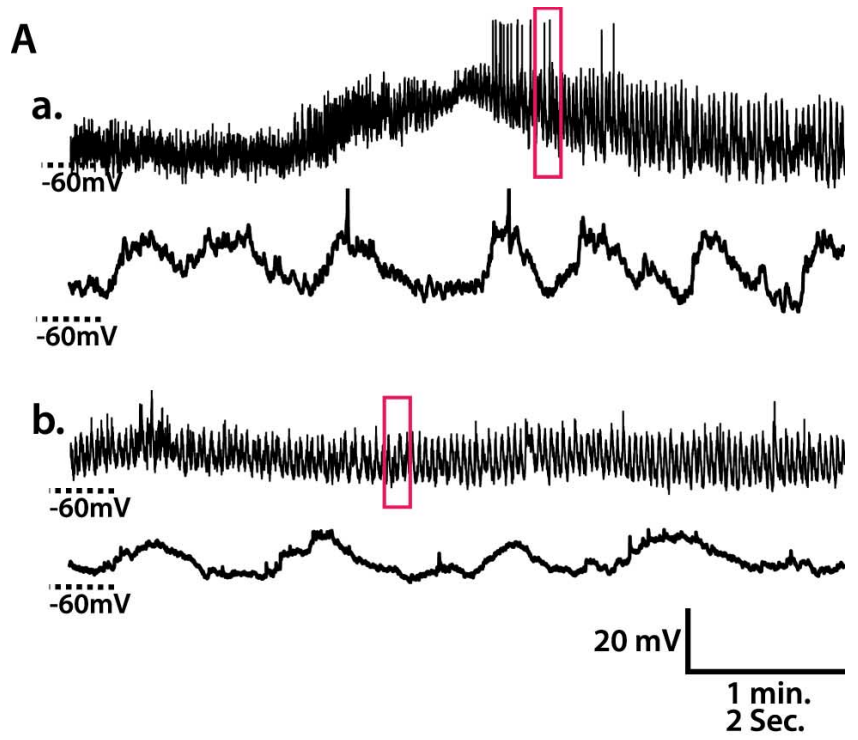

B

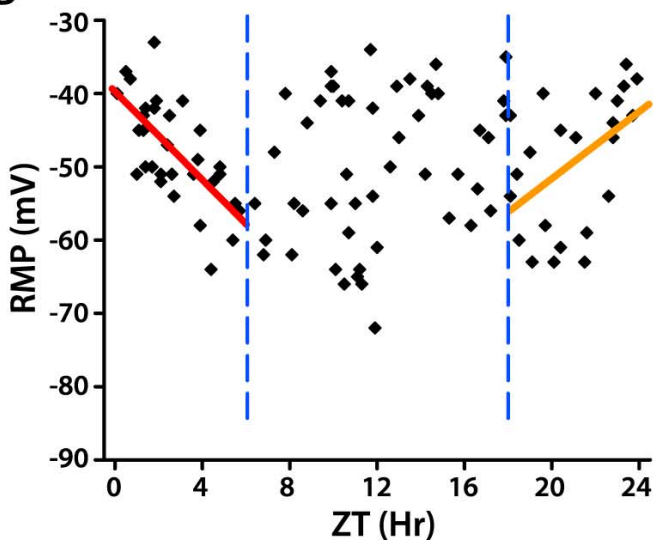

Figure 7. WT $S L N_{v} R M P$ is most depolarized near lights-on. $A$, Examples of whole-cell recordings on $L_{\mathrm{N}} \mathrm{s}, \boldsymbol{a}, \boldsymbol{b}$, Top panels are 5 min recordings, and bottom panels are the enlargement of the $10 \mathrm{~s}$ traces indicated in the red box of 5 min traces. $\boldsymbol{a}$, An example of an $\mathrm{sLN} \mathrm{v}_{\mathrm{v}}$ that exhibits spontaneous APs. $\boldsymbol{b}$, An example of an $s L N_{v}$ that exhibits no APs. $\boldsymbol{B}$, Scatter plot of WT $s \mathrm{LN}_{\mathrm{v}}$ RMP versus time of day in LD. The relationship between RMP and time of day is analyzed using linear regression analysis. From ZT0 to ZT6, RMP becomes hyperpolarized (red line, $p<0.0001$ ), and from ZT18 to ZT24 it becomes depolarized (orange line, $p<0.05$ ), thus indicating a peak depolarization around lights-on. Two blue vertical dashed lines mark ZT6 and ZT18, respectively.

membrane resistance exhibit no significant changes over $24 \mathrm{~h}$ in $\mathrm{DD}$. This absence of rhythmic changes in $\mathrm{ILN}_{\mathrm{v}}$ membrane excitability in DD, when transcriptional feedback oscillation ceases in $\operatorname{lLN}_{\mathrm{v}} \mathrm{s}$ but continues in other clock neurons, supports the conclusion that the rhythmic changes occurring in LD are driven cellautonomously by the circadian clock of $\mathrm{LLN}_{\mathrm{v}} \mathrm{s}$ themselves.

\section{Regulation of $s \mathrm{LN}_{\mathrm{v}}$ membrane excitability in $\mathrm{LD}$}

Although the specific function of $\operatorname{LN}_{\mathrm{v}}$ versus $\mathrm{SLN}_{\mathrm{v}}$ clock neurons remains unclear, it has been suggested that the $\mathrm{sLN}_{\mathrm{v}} \mathrm{s}$ are particularly important pacemakers of locomotor activity (Grima et al., 2004). Although the size and location of $\mathrm{sLN}_{\mathrm{v}} \mathrm{s}$ make it much more difficult to obtain stable recordings, we obtained whole-cell recordings of $103 \mathrm{sLN}_{\mathrm{v}} \mathrm{s}$ in $\mathrm{LD}$, each from one individual fly. As shown in Figure $7 A$, the RMP of some $\operatorname{sLN}_{\mathrm{v}} \mathrm{s}$ exhibit $3 \sim 10 \mathrm{mV}$ oscillation, like $\mathrm{lLN}_{\mathrm{v}} \mathrm{s}$. Few $\mathrm{sLN}_{\mathrm{v}} \mathrm{s}$ (18 of 103) fire spontaneous APs, and small synaptic transmission-like transients with an amplitude of $2-5 \mathrm{mV}$ are found in $47 \%$ of $s \mathrm{LN}_{\mathrm{v}} \mathrm{s}$. The relationship of $s N_{v}$ RMP to time of day in LD is plotted in Figure 7B. sLN $\mathrm{v}_{\mathrm{v}} \mathrm{RMP}$ is highest around lights-on. From ZT0 to ZT6, sLN ${ }_{v}$ RMP exhibited a significant decrease (linear regression, $p<0.0001$ ), and from ZT18 to ZT24, sLN $\mathrm{v}_{\mathrm{v}} \mathrm{RMP}$ showed a significant increase (linear regression, $p=0.018$ ). However, from ZT6 to ZT18, the distribution of $\mathrm{sLN}_{\mathrm{v}} \mathrm{RMP}$ had no significant nonzero slope (linear regression, $p>0.05$ ) and was more variable. The peak of $\mathrm{sLN}_{\mathrm{v}}$ depolarization at lights-on is consistent with the temporal pattern of ILN $_{\mathrm{v}}$ RMP.

\section{Discussion}

In this study, we investigated the temporal regulation of membrane excitability of PDF-expressing $\mathrm{LN}_{\mathrm{v}}$ clock neurons. We characterized RMP and AP firing patterns in $\operatorname{lLN}_{\mathrm{v}} \mathrm{s}$, and demonstrate that $\mathrm{ILN}_{\mathrm{v}}$ membrane excitability oscillates as a function of time of day in LD. We showed furthermore that this oscillation requires a functional circadian oscillator, as it is abolished in per $^{\circ}$ flies. This requirement could be cell autonomous to the $\operatorname{lLN}_{\mathrm{v}} \mathrm{s}$, as suggested by the fact that the oscillation is abolished in DD, a condition in which $\mathrm{ILN}_{\mathrm{v}}$, but not other clock neuron, transcriptional oscillation ceases. We found that $\mathrm{sLN}_{\mathrm{v}} \mathrm{RMP}$ is most depolarized near lights-on.

$\mathrm{ILN}_{\mathrm{v}}$ spontaneous activity is somewhat variable, with $33 \%$ of cells silent in the light phase and 38\% in the dark in LD. That, overall, $35 \%$ of $1 \mathrm{LN}_{\mathrm{v}} \mathrm{s}$ are silent in $\mathrm{LD}$ is slightly higher but still comparable with mammalian clock neurons in the suprachiasmatic nucleus (SCN) (Walsh et al., 1992; Kim and Dudek, 1993). Despite the $35 \%$ of silent $\operatorname{lLN}_{\mathrm{v}} \mathrm{s}$, the average AP firing frequency and the percentage of $1 \mathrm{LN}_{\mathrm{v}} \mathrm{s}$ that fire APs show a circadian temporal pattern similar to that in mammalian SCN, which is consistent with linear regression analysis of the scatter plot of individual cells (Fig. $4 \mathrm{~B}$ ). Interestingly, in contrast to the cyclic changes in $1 \mathrm{LN}_{\mathrm{v}}$ RMP, membrane resistance and AP firing rate decrease from dawn to dusk in the day but stay low during the night in LD (Fig. 4 B, C). The biophysical basis for this uncoupling of RMP from AP firing rate and membrane resistance at night is not clear, but presumably reflects differential modulation of particular ion channel subtypes. This issue bears further investigation in the future using physiological, pharmacological, and genetic techniques.

$\mathrm{ILN}_{\mathrm{v}} \mathrm{s}$ exhibit two sizes of APs, with the small APs approximately half the amplitude of the large APs (Fig. 1C). Gap junction inhibitors reduce the frequency of small APs and alter the normal firing rate of large APs, suggesting that gap junctions are involved in modulating membrane excitability of $\mathrm{LLN}_{\mathrm{v}} \mathrm{s}$. This is consistent with observations of cockroach lateral neurons (Schneider and Stengl, 2006), where gap junction blockers also influence spontaneous AP firing rate, and clock neurons in mammalian SCN (Colwell, 2000; Shinohara et al., 2000; Long et al., 2005; Rash et al., 2007). However, none of the three gap junction blockers had any effect on the amplitude of either large or small APs, thus making it highly unlikely that APs generated in coupled cells are propagating passively to the site of recording at the soma through gap junctions. That cutting the POT removes small APs strongly supports that small APs originate in the contralateral optic lobes. This raises the interesting possibility that the ipsilateral and contralateral arbors of individual $\mathrm{LLN}_{\mathrm{v}} \mathrm{s}$ can process information independently.

We demonstrated that WT $\mathrm{ILN}_{\mathrm{v}}$ membrane excitability is modulated as a function of time of day. In particular, in the light phase of LD, ILN $\mathrm{v}_{\mathrm{v}}$ RMP becomes more hyperpolarized from dawn to dusk, as the AP firing rate and $\mathrm{lLN}_{\mathrm{v}}$ membrane resistance decrease. In the dark phase, ILN $_{\mathrm{v}}$ RMP becomes more depolar- 
ized from the beginning to the end of the night, whereas the AP firing rate and membrane resistance stay relatively stable (Fig. 4). This continuous cyclical modulation of WT $\mathrm{ILN}_{\mathrm{v}}$ RMP is consistent with previous coarse time-scale studies demonstrating that $\mathrm{ILN}_{\mathrm{v}} \mathrm{RMP}$ is more depolarized in the early morning and more hyperpolarized in the early night (Park and Griffith, 2006; Sheeba et al., 2008). This temporal modulation is almost completely abolished in $\operatorname{per}^{0}$ flies (Fig. 5), thus demonstrating for the first time its dependence on an intact transcriptional feedback oscillator. Although this is consistent with an autonomous requirement for transcriptional feedback oscillation in the $\operatorname{lLN}_{\mathrm{v}} \mathrm{s}$ themselves, because per $^{0}$ flies lack transcriptional feedback oscillation in all neurons, this could be a circuit-based phenomenon. To further address this question, we examined the modulation of WT ILN $_{v}$ excitability on the first day in DD conditions (DD-D1), in which transcriptional oscillation in $\operatorname{lLN}_{\mathrm{v}} \mathrm{s}$, but not in any other clock neurons, is abolished (Yang et al., 1998; Veleri et al., 2003; Grima et al., 2004; Nitabach et al., 2006; Wu et al., 2008). The absence of oscillatory modulation of $\mathrm{LLN}_{\mathrm{v}}$ excitability in DD-D1 further supports the conclusion that transcription feedback oscillation is required cell-autonomously in the $\operatorname{lLN}_{\mathrm{v}} \mathrm{s}$, and the transcriptional feedback oscillator directly modulates $\operatorname{lLN}_{\mathrm{v}}$ membrane excitability at the cellular level. The apparent difference between our demonstration of an absence of dependence of electrical excitability on time of day on DD-D1, and Sheeba et al.'s (2008) demonstration of presence of a dependence of electrical excitability on time of day on DD-D14 actually makes sense in light of what is known about the response of $\operatorname{lLN}_{\mathrm{v}}$ s to constant darkness. Whereas $\mathrm{sLN}_{\mathrm{v}} \mathrm{s}$ and other clock neurons maintain their cellular rhythmicity immediately after transition from LD to DD conditions, the $\mathrm{ILN}_{\mathrm{v}} \mathrm{s}$ immediately lose their cellular rhythms with transition into DD (Yang and Sehgal, 2001; Shafer et al., 2002), and only regain cellular rhythmicity after several days in DD (Peng et al., 2003; Stoleru et al., 2004). This immediate loss of $\mathrm{ILN}_{\mathrm{v}}$ cellular rhythmicity followed by resumption several days later perfectly accounts for the fact that we observed no rhythm of $\mathrm{ILN}_{\mathrm{v}}$ electrical excitability on DD-D1 whereas Sheeba et al. (2008) observed such a rhythm on DD-D14.

Either $p d f$ gene mutation, $\mathrm{LN}_{\mathrm{v}}$ ablation, or $\mathrm{LN}_{\mathrm{v}}$ electrical silencing severely disrupts free-running locomotor rhythms (Renn et al., 1999; Nitabach et al., 2002), indicating that PDF-expressing $\mathrm{LN}_{\mathrm{v}} \mathrm{s}$ play a critical role in controlling circadian behavior. Both $s L N_{v} s$ and $\operatorname{lLN}_{v} s$ express PDF. However, previous studies suggest that of these two cell groups it is the $\mathrm{sLN}_{\mathrm{v}} \mathrm{s}$ that are most important for controlling free-running rhythms in DD. In particular, restoration of per gene expression in $\mathrm{sLN}_{\mathrm{v}} \mathrm{s}$, but not $\mathrm{lLN}_{\mathrm{v}} \mathrm{s}$ can rescue free-running circadian rhythmicity in per $^{\circ}$ null mutant flies (Grima et al., 2004). Furthermore, as mentioned above, $\mathrm{lLN}_{\mathrm{v}}$ transcriptional feedback oscillation cease in DD (Yang and Sehgal, 2001; Shafer et al., 2002). The $\operatorname{lLN}_{\mathrm{v}} \mathrm{s}$ have extensive dendritic arborizations in the medulla and project to contralateral $1 \mathrm{LN}_{\mathrm{v}} \mathrm{s}$ through the posterior optic tracts, thus enabling enable them to potentially receive light information and synchronize the two hemispheres (Helfrich-Forster and Homberg, 1993; Kaneko and Hall, 2000). The $\mathrm{ILN}_{\mathrm{v}} \mathrm{s}$ also project to the accessory medulla (aMe), where the $\mathrm{LN}_{\mathrm{v}} \mathrm{s}$ have short dendrites that may be postsynaptic to lLN $_{\mathrm{v}}$ projections (Helfrich-Forster et al., 2007). Although the functional role of $\mathrm{LLN}_{\mathrm{v}} \mathrm{s}$ in circadian rhythms is mostly unknown, it is reasonable to speculate based on this anatomy that $1 \mathrm{LN}_{\mathrm{v}}$ s relay environmental light information to the $\mathrm{LN}_{\mathrm{v}} \mathrm{s}$ via synapses formed in aMe. We thus propose that circadian modulation of $\mathrm{ILN}_{\mathrm{v}}$ membrane excitability in LD gates the transfer of entrain- ing light information to the $s \mathrm{LN}_{\mathrm{v}} \mathrm{s}$, which more directly control circadian locomotor rhythms.

Because $\mathrm{sLN}_{\mathrm{v}} \mathrm{s}$ are thought to be pacemakers of the neural circuit controlling circadian rhythms (Park et al., 2000; Yang and Sehgal, 2001; Grima et al., 2004), it is critical to understand how membrane excitability of $s \mathrm{LN}_{\mathrm{v}} \mathrm{s}$ is regulated, and how $\mathrm{sLN}_{\mathrm{v}}$ membrane excitability is transformed to signals that control downstream neurons, and, ultimately, circadian locomotor activity. We recorded $103 \mathrm{SLN}_{\mathrm{v}} \mathrm{s}$ in LD and found that $\mathrm{sLN} \mathrm{v}_{\mathrm{v}} \mathrm{RMP}$ is most depolarized around lights-on, with greater variability around lights-off (Fig. 7B). The two linear regression fits were selected to span the $6 \mathrm{~h}$ just before, and just after, lights-on (ZT0, ZT24). $s L N_{v}$ RMP exhibits significant trends of depolarization before lights-on and hyperpolarization after lights-on. It is clear that during the $12 \mathrm{~h}$ surrounding lights-off (ZT6-ZT18), there is much more variability in $\mathrm{sLN}_{\mathrm{v}} \mathrm{RMP}$. This difference makes some sense, as $\mathrm{sLN}_{\mathrm{v}} \mathrm{s}$ are considered to be the "morning" cells that drive the increase in locomotor activity that anticipates lights-on (Grima et al., 2004; Stoleru et al., 2004). This would be consistent with an increase in $\mathrm{sLN}_{\mathrm{v}}$ electrical excitability in the hours preceding lights-on, and a decrease after lights-on, as we clearly observed. Because the $s \mathrm{LN}_{\mathrm{v}} \mathrm{s}$ are not thought to be important for driving the increase in locomotor activity that anticipates lightsoff (ZT12) (Grima et al., 2004; Stoleru et al., 2004), it is perhaps not surprising that there are no consistent trends in $\mathrm{sLN}_{\mathrm{V}}$ electrical excitability surrounding lights-off (ZT12). The reason $\mathrm{sLN}_{\mathrm{v}} \mathrm{s}$ exhibit little spontaneous activity in this brain explant preparation is not clear, but it may reflect technical issues relating to the extremely small size of their soma. Previous studies indicate that the peak of anti-PDF staining in the $\operatorname{sLN}_{\mathrm{v}}$ nerve terminals is around lights-on, and the trough is around lights-off (Park et al., 2000; Nitabach et al., 2006). It is thus possible that the trough of PDF accumulation around lights-off represents depletion of the releasable pool of PDF starting in the early day, when $\mathrm{SLN}_{\mathrm{v}} \mathrm{s}$ are most depolarized.

Our study, and that by Sheeba et al. (2008), demonstrate a dependence of $1 \mathrm{LN}_{\mathrm{V}}$ electrical excitability on time of day in LD and after many days in DD, respectively. Our results go beyond those of Sheeba et al. (2008) in a number of important ways, including demonstration that the daily rhythm of $1 \mathrm{LN}_{\mathrm{V}}$ electrical excitability depends on a functional cellular circadian oscillator, demonstration of a daily rhythm of electrical excitability in the $\mathrm{sLN}_{\mathrm{V}}$ pacemaker subset of clock neurons, and elucidation of the cellular origin of the bimodal distribution of sizes of $\mathrm{ILN}_{\mathrm{V}}$ APs. Future studies will determine the daily rhythms of electrical excitability of other functionally and anatomically identifiable subsets of fly clock neurons.

\section{References}

Colwell CS (2000) Rhythmic coupling among cells in the suprachiasmatic nucleus. J Neurobiol 43:379-388.

de Jeu M, Hermes M, Pennartz C (1998) Circadian modulation of membrane properties in slices of rat suprachiasmatic nucleus. NeuroReport 9:3725-3729.

Green DJ, Gillette R (1982) Circadian rhythm of firing rate recorded from single cells in the rat suprachiasmatic brain slice. Brain Res 245:198-200.

Grima B, Chelot E, Xia R, Rouyer F (2004) Morning and evening peaks of activity rely on different clock neurons of the Drosophila brain. Nature 431:869-873.

Groos G, Hendriks J (1982) Circadian rhythms in electrical discharge of rat suprachiasmatic neurones recorded in vitro. Neurosci Lett 34:283-288.

Hardin PE (2005) The circadian timekeeping system of Drosophila. Curr Biol 15:R714-R722.

Harmer SL, Panda S, Kay SA (2001) Molecular bases of circadian rhythms. Annu Rev Cell Dev Biol 17:215-253. 
Helfrich-Forster C (2002) The circadian system of Drosophila melanogaster and its light input pathways. Zoology (Jena) 105:297-312.

Helfrich-Forster C (2003) The neuroarchitecture of the circadian clock in the brain of Drosophila melanogaster. Microsc Res Tech 62:94-102.

Helfrich-Forster C, Homberg U (1993) Pigment-dispersing hormoneimmunoreactive neurons in the nervous system of wild-type Drosophila melanogaster and of several mutants with altered circadian rhythmicity. J Comp Neurol 337:177-190.

Helfrich-Forster C, Tauber M, Park JH, Muhlig-Versen M, Schneuwly S, Hofbauer A (2000) Ectopic expression of the neuropeptide pigmentdispersing factor alters behavioral rhythms in Drosophila melanogaster. J Neurosci 20:3339-3353.

Helfrich-Forster C, Shafer OT, Wulbeck C, Greishaber E, Rieger D, Taghert P (2007) Development and morphology of the clock-gene-expressing lateral neurons of Drosophila melanogaster. J Comp Neurol 500:47-70.

Itri JN, Michel S, Vansteensel MJ, Meijer JH, Colwell CS (2005) Fast delayed rectifier potassium current is required for circadian neural activity. Nat Neurosci 8:650-656.

Kaneko M, Hall JC (2000) Neuroanatomy of cells expressing clock genes in Drosophila: transgenic manipulation of the period and timeless genes to mark the perikarya of circadian pacemaker neurons and their projections. J Comp Neurol 422:66-94.

Kim YI, Dudek FE (1993) Membrane properties of rat suprachiasmatic nucleus neurons receiving optic nerve input. J Physiol (Lond) 464:229-243.

Konopka RJ, Benzer S (1971) Clock mutants of Drosophila melanogaster. Proc Natl Acad Sci USA 68:2112-2116.

Kuhlman SJ, McMahon DG (2004) Rhythmic regulation of membrane potential and potassium current persists in SCN neurons in the absence of environmental input. Eur J Neurosci 20:1113-1117.

Long MA, Jutras MJ, Connors BW, Burwell RD (2005) Electrical synapses coordinate activity in the suprachiasmatic nucleus. Nat Neurosci 8:61-66.

Nitabach MN, Taghert PH (2008) Organization of the Drosophila circadian control circuit. Curr Biol 18:R84-R93.

Nitabach MN, Blau J, Holmes TC (2002) Electrical silencing of Drosophila pacemaker neurons stops the free-running circadian clock. Cell 109:485-495.

Nitabach MN, Sheeba V, Vera DA, Blau J, Holmes TC (2005) Membrane electrical excitability is necessary for the free-running larval Drosophila circadian clock. J Neurobiol 62:1-13.

Nitabach MN, Wu Y, Sheeba V, Lemon WC, Strumbos J, Zelensky PK, White BH, Holmes TC (2006) Electrical hyperexcitation of lateral ventral pacemaker neurons desynchronizes downstream circadian oscillators in the fly circadian circuit and induces multiple behavioral periods. J Neurosci 26:479-489.

Park D, Griffith LC (2006) Electrophysiological and anatomical characterization of PDF-positive clock neurons in the intact adult Drosophila brain. J Neurophysiol 95:3955-3960.

Park JH, Helfrich-Forster C, Lee G, Liu L, Rosbash M, Hall JC (2000) Differential regulation of circadian pacemaker output by separate clock genes in Drosophila. Proc Natl Acad Sci USA 97:3608-3613.
Peng Y, Stoleru D, Levine JD, Hall JC, Rosbash M (2003) Drosophila freerunning rhythms require intercellular communication. PLoS Biol 1:e13.

Pennartz CM, de Jeu MT, Bos NP, Schaap J, Geurtsen AM (2002) Diurnal modulation of pacemaker potentials and calcium current in the mammalian circadian clock. Nature 416:286-290.

Pitts GR, Ohta H, McMahon DG (2006) Daily rhythmicity of largeconductance $\mathrm{Ca}^{2+}$-activated $\mathrm{K}^{+}$currents in suprachiasmatic nucleus neurons. Brain Res 1071:54-62.

Rash JE, Olson CO, Pouliot WA, Davidson KG, Yasumura T, Furman CS, Royer S, Kamasawa N, Nagy JI, Dudek FE (2007) Connexin36 vs connexin32, "miniature" neuronal gap junctions, and limited electrotonic coupling in rodent suprachiasmatic nucleus. Neuroscience 149:350-371.

Renn SC, Park JH, Rosbash M, Hall JC, Taghert PH (1999) A pdf neuropeptide gene mutation and ablation of PDF neurons each cause severe abnormalities of behavioral circadian rhythms in Drosophila. Cell 99:791-802.

Rosato E, Tauber E, Kyriacou CP (2006) Molecular genetics of the fruit-fly circadian clock. Eur J Hum Genet 14:729-738.

Schneider NL, Stengl M (2006) Gap junctions between accessory medulla neurons appear to synchronize circadian clock cells of the cockroach Leucophaea maderae. J Neurophysiol 95:1996-2002.

Shafer OT, Rosbash M, Truman JW (2002) Sequential nuclear accumulation of the clock proteins period and timeless in the pacemaker neurons of Drosophila melanogaster. J Neurosci 22:5946-5954.

Shafer OT, Helfrich-Forster C, Renn SC, Taghert PH (2006) Reevaluation of Drosophila melanogaster's neuronal circadian pacemakers reveals new neuronal classes. J Comp Neurol 498:180-193.

Sheeba V, Gu H, Sharma VK, O’Dowd DK, Holmes TC (2008) Circadianand light-dependent regulation of resting membrane potential and spontaneous action potential firing of Drosophila circadian pacemaker neurons. J Neurophysiol 99:976-988.

Shinohara K, Hiruma H, Funabashi T, Kimura F (2000) GABAergic modulation of gap junction communication in slice cultures of the rat suprachiasmatic nucleus. Neuroscience 96:591-596.

Stoleru D, Peng Y, Agosto J, Rosbash M (2004) Coupled oscillators control morning and evening locomotor behaviour of Drosophila. Nature 431:862-868.

Veleri S, Brandes C, Helfrich-Forster C, Hall JC, Stanewsky R (2003) A selfsustaining, light-entrainable circadian oscillator in the Drosophila brain. Curr Biol 13:1758-1767.

Walsh IB, van den Berg RJ, Marani E, Rietveld WJ (1992) Spontaneous and stimulated firing in cultured rat suprachiasmatic neurons. Brain Res 588:120-131.

Wu Y, Cao G, Nitabach MN (2008) Electrical silencing of PDF neurons advances the phase of non-PDF clock neurons in Drosophila. J Biol Rhythms 23:117-128.

Yang Z, Sehgal A (2001) Role of molecular oscillations in generating behavioral rhythms in Drosophila. Neuron 29:453-467.

Yang Z, Emerson M, Su HS, Sehgal A (1998) Response of the timeless protein to light correlates with behavioral entrainment and suggests a nonvisual pathway for circadian photoreception. Neuron 21:215-223. 